\title{
Article
}

\section{Paediatric intensive care nurses' decision-making around gastric residual volume measurement}

Tume, Lyvonne Nicole, Latten, Lynne and Kenworthy, Lindsay

Available at https://clok.uclan.ac.uk/18623/

Tume, Lyvonne Nicole orcid iconORCID: 0000-0002-2547-8209, Latten, Lynne and Kenworthy, Lindsay (2017) Paediatric intensive care nurses' decisionmaking around gastric residual volume measurement. Nursing in Critical Care, 22 (5). pp. 293-297. ISSN 1362-1017

It is advisable to refer to the publisher's version if you intend to cite from the work. http://dx.doi.org/10.1111/nicc.12304

For more information about UCLan's research in this area go to http://www.uclan.ac.uk/researchgroups/ and search for <name of research Group>.

For information about Research generally at UCLan please go to http://www.uclan.ac.uk/research/

All outputs in CLoK are protected by Intellectual Property Rights law, including Copyright law. Copyright, IPR and Moral Rights for the works on this site are retained by the individual authors and/or other copyright owners. Terms and conditions for use of this material are defined in the policies page.

\section{CLoK}

Central Lancashire online Knowledge www.clok.uclan.ac.uk

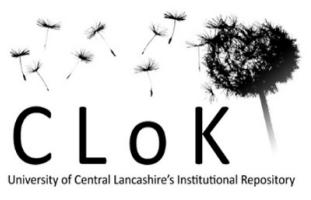


Paediatric intensive care nurses' decision-making around gastric residual volume measurement

\section{Abstract}

\section{Background}

Measuring gastric residual volume (GRV) to guide enteral feeding is a common nursing practice in intensive care units, yet little evidence supports this practice. In addition, this practice has been shown to potentially contribute to inadequate energy delivery in intensive care, which remains a problem in critically ill children.-

\section{Aims}

We aimed to explore paediatric intensive care nurses' decision-making surrounding this practice.

\section{Methods}

A cross-sectional electronic survey in a single mixed general and cardiac surgical PICU in the UK.

\section{Results}

The response rate was 59\% (91/154) and responding nurses were experienced, with a mean PICU experience of 10.5 years (SD 8.09). The three main reasons for stopping or withholding enteral feeds were: the volume of gastric residual volume obtained (67\%), the appearance of this gastric aspirate (40\%) and the overall clinical condition of the child (23\%). Most 
nurses reported checking GRV primarily to determine 'feed tolerance' (97\%) as well as confirming feeding tube position (94\%). Nurses' perceived harms from high GRV were: the risk of pulmonary aspiration (44\%), malabsorption of feeds (20\%) and the risk of vomiting (19\%). GRV was measured frequently in this PICU, with 58\% measuring GRV before every feed, $27 \%$ measuring 4 hourly and $17 \%$ measuring 6 hourly.

The majority of nurses (84\%) stated they would be worried or very worried if they could not measure GRV routinely.

\section{Conclusions}

PICU nurses' decision-making surrounding initiating and withholding enteral feeds, and determining 'feed tolerance' remains heavily based on GRV. PICU nurses' have significant fears around patient harm if they do not measure GRV routinely.

\section{Relevance to clinical practice}

This nursing practice is likely to be one of the factors that impair the delivery of enteral nutrition in critically ill children and as such its validity and usefulness needs to be challenged and studied in future research. 


\section{INTRODUCTION}

Underfeeding remains a constant problem in paediatric intensive care units (PICUs). A large international point prevalence study showed only $37 \%$ of children received their prescribed energy intake, and that it took nearly 12 days to achieve $90 \%$ of their calorie target (Mepta et al 2012). It is a common nursing practice to assess patient's 'tolerance' to enteral nutrition (EN) by measuring gastric residual volume (GRV) (Tume et al 2013; Valla et al 2015). GRV is known to be a significant factor in the decision to stop or hold enteral nutrition (Leong et al 2013). Interruptions to EN in the PICU are known to be one of the biggest barriers to delivering adequate nutrition (Mepta et al 2010; Leong et al 2013). Therefore, we wanted to explore PICU nurses' decision-making around the practice of GRV measurement.

\section{METHODS}

A cross-sectional electronic survey was conducted in a single mixed medical-surgical PICU in North West England. The survey instrument was developed by two PICU nurses and a dietician (LK,LNT, LL), as no previous instruments existed, to explore questions that had arisen from a previous study (Tume et al 2017). This 20 item instrument was tested on 10 nurses (both junior and senior) for clarity and face validity and changes were made to improve question clarity (Figure 1 Survey Instrument). The survey was input into electronic

software (Survey Monkey ${ }^{\mathrm{TM}}$ ) and tested again by an independent PICU nurse. After registration by the hospital (NHS trust) as audit (Reference No. 5339) it was sent out to all nurses $(n=152)$ and assistant practitioners $(n=2)$ in the PICU in August 2016. Consent was 
implied by return of the survey. Three reminders were sent, one week apart, to maximise response rates with a target response rate of $70 \%$.

Inclusion criteria: Clinical nurses or assistant nursing practitioners who are working on PICU and make decisions around feeding. Exclusion criteria: non clinical nurses, nurses not working in bedside nursing roles and bank or agency staff.

\section{Study Setting and standard practice}

The PICU is a 23 bed intensive care unit which admits around 1000 children a year aged $0-$ 17 years. The unit has a separate 15 bed high dependency unit staffed by different nurses; not included in the study. It is a mixed cardiac surgical and general intensive care unit and $86 \%$ of the patients receive invasive ventilation (PICANET 2016). The nurse to patient ratio is $1: 1$ for all invasively ventilated children and $52 \%$ of the nursing staff have a specialised post-graduate PICU nursing course. The unit has a detailed feeding protocol requiring 4-5 hourly GRV measurement and withholding feeds if this volume exceeds $5 \mathrm{ml} / \mathrm{kg}$ to a maximum of $300 \mathrm{ml}$. The unit is proactive in starting enteral feeding (guidelines state within 6 hours after PICU admission, unless contraindications exist). The unit is supported by a dedicated dietician who reviews patients daily and does weekly 'nutrition rounds' with a gastroenterologist. Our feeding protocol includes routine GRV assessment to assess 'tolerance' to enteral nutrition. Feeding delivery method is most commonly bolus gravity feeds in infants and continuous pump feeds in older children, but this decision is left up to the registered nurse.

\section{Data analysis}

In this small exploratory study, data was exported from a CSV file in Survey Monkey into Microsoft Excel and IBM SPSS version 22 for further analysis. Data was analysed primarily 
descriptively, but inferential analysis (Chi square) was used to determine whether nurses' experience or speciality education impacted on key outcomes (categorical variables). $\mathrm{P}$ values $<0.05$ were considered significant and two tailed test were used. Free text responses in this survey, were to direct questions and so analysed by simple thematic analysis (Burnard et al 2008). Responses were categorised independently by LT and LK, who then met to agree the categorisation and groupings where any discrepancies were discussed and agreed.

\section{RESULTS}

The response rate was $58 \%(90 / 154)$ and responding nurses were experienced, with a mean PICU experience of 10.5 years (SD 8.09). 76\% nurses had a specialist PICU nursing qualification. $63 \%$ were staff nurses, $27 \%$ senior staff nurses, $8 \%$ sisters or charge nurses and $2 \%$ assistant nursing practitioners. PICU nurses perceived their role in initiating, delivering and evaluating enteral nutrition as all highly important (Figure 2). The three highest perceived barriers to delivering adequate nutrition in this PICU were: Fluid restriction (52\%, specifically in cardiac children), nurses' education, attitudes and knowledge (33\%) and fasting for procedures (33\%). The three main reasons for stopping or withholding enteral feeds were: the volume of gastric residual volume obtained (67\%), the appearance of this gastric aspirate (40\%) and the overall clinical condition of the child (23\%).

Most nurses reported checking GRV primarily to determine 'feed tolerance' (97\%) as well as confirming feeding tube position (94\%). Nurses' perceived harms from high GRV were: the risk of pulmonary aspiration (44\%), malabsorption of feeds (20\%) and the risk of vomiting (19\%) (Table 1). GRV was measured frequently in this PICU, with 58\% measuring GRV before every feed, $27 \%$ measuring 4 hourly and $17 \%$ measuring 6 hourly. 
The majority of nurses (84\%) stated they would be worried or very worried if they could not measure GRV routinely, with their biggest concerns being: not able to measure feed 'tolerance' (55\%), not being able to confirm feeding tube position (32\%) and the risk of vomiting and aspiration (27\%). Most nurses were aware of other ways they could assess feed tolerance, citing bowel movements (62\%), abdomen appearance (59\%), vomiting (38\%), the presence of bowel sounds (25\%), serum lactate level (21\%) and signs of patient discomfort (16\%) (Figure 3). When asked to consider NOT routinely measuring GRV as part of a research study, $50 \%$ of nurses were broadly negative, $43 \%$ were broadly positive (so long as clear guidance was provided) and $3 \%$ were indifferent. The majority of nurses who were positive towards a trial were significantly more experienced $(p=<0.000)$ and had a PICU nursing qualification $(p=<0.000)$.

\section{DISCUSSION}

This is the first study to our knowledge to attempt to explore PICU nurses' decision making around the practice of GRV measurement in the paediatric ICU. Other studies involved neonatal intensive care nurses (Hodges and Vincent 1993) or adult intensive care (Admad et al 2012). The practice of routine GRV measurement is increasingly being questioned across critical care as a whole (in neonates, children and adults) (Kuppinger et al 2013, Parker et al 2015; Li et al 2014; Bollineni et al 2011, Parish et al 2008). In a multicentre observational study in 19 adult ICUs in France, Quenot et al (2010) showed that just by measuring GRV, the risk of delivering inadequate energy goals increased by $38 \%$.

We found nurses were very concerned about the risk of aspiration if they could not measure GRV. Others have also found that GRV featured heavily in healthcare professionals' beliefs that measuring GRV mitigates the perceived risk of pulmonary aspiration in mechanically 
ventilated patients (Ahmand et al 2012), but this risk remains unquantified (McClave et al 2005). In adult intensive care trials, accepting a higher GRV ( $500 \mathrm{ml}$ compared to $200 \mathrm{ml}$ ) (Montejo et al 2010) or not measuring GRV at all (Poulard et al 2010; Reignier et al 2013; Ozen et al 2016) did not adversely affect patient outcomes of ventilator associated pneumonia(VAP) or gastrointestinal complications, however did it increase the achievement of the patient's energy goals, and increase calorie delivery.

In this survey we found GRV was the main reason perceived by nurses for stopping enteral feeding. Interruptions to feeding have been cited by others as probably the biggest factor in delivering suboptimal nutrition in critically ill patients (Mehta et al 2010; Bockenkamp et al 2009). Nurses said they predominantly used GRV to determine feed 'tolerance', but the ability of this measurement to do this is questionable. Despite the widespread prevalence of this practice (Tume et al 2012, Valla et al 2015; Ahmad et al 2012) GRV has not been show to correlate with enteral feeding tolerance (McCLave et al 2002). In addition, the measurement of GRV is frequently inaccurate due to the position of the feeding tube in the stomach, patient position, the feeding method, the technique of aspiration and tube and syringe sizes used (McClave et al 2005; Bartlett-Ellis et al 2015; Elke et al 2015). Compounding this uncertainty is what volume constitutes an 'acceptable' level of GRV. In our study nurses main cited reasons for impaired enteral feeding on the ICU were consistent with what others have found in terms of fluid restriction (Tume et al 2013; Floh et al 2016) and fasting for procedures (Mehta et al 2010; Bockenkamp et al 2009). It was notable however, that nurses themselves perceived that inadequate knowledge, education and attitudes impacted on enteral feeding. Marik (2014) reviewed the evidence for 
commonly believed myths and misconceptions held by ICU staffs that contribute to underfeeding.

We found some confusion in nurses thinking surrounding confirming feeding tube position. Although a legal requirement in the UK to avoid misplaced tubes and inadvertent feeding into the airways, (National Patient Safety Agency 2011) nurses cited GRV was used to confirm tube position. However, the volume required to test gastric aspirate for ph. is very small, the whole stomach contents (GRV) does not have to be aspirated to do this, and yet it seemed this is what many believed was required. This is an area for educational intervention.

When asked to consider other indicators that could be used to assess the tolerance of enteral feeding, most (but not all) PICU nurses could cite other signs. This demonstrates that lack of any consistently valid method to assess feed tolerance in all critically ill patients, and therefore the reliance on, and the overestimation of, the ability of a fairly simplistic indicator, such as GRV, as a measurement to do this.

At least half of the nurses were very worried and gave negative responses about the idea of not measuring GRV as part of a research study. This is important to know when considering the design of any future research on this topic, as it may impact significantly on the compliance with study protocols. It is evident that PICU nurses' beliefs around GRV are strongly held and there would need to be considerable work done to overcome these. There are a number of limitations that need acknowledgment, including those biases associated with self-report surveys including selection bias, self-report bias, confounding, lack of generalizability, and no means of data verification from participants. It is a single 
centre survey and there may be unit-specific views that do not reflect PICU nurses in other units. In addition, we achieved a lower than expected response rate of 59\%. Despite these limitations, this is the first study to attempt to explore in more detail PICU nurses' decisionmaking around this common practice.

\section{CONCLUSIONS}

Nurses play a vital role in the delivery of enteral nutrition for critically ill children. Their decision- making surrounding initiating and withholding enteral feeds, and determining 'feed tolerance' is heavily based on GRV, yet this practice is not supported by evidence. Most nurses cited the fear of pulmonary aspiration was their main concern if GRV was not measured. Further research needs to explore this beyond a single UK PICU, and researchers need to understand nurses' views if future trials to avoid this practice are planned.

\section{What is known about this topic?}

- Routine GRV measurement is a widespread nursing practice

- Both the accuracy and interpretation of GRV measurement however is not based on evidence and may impair the delivery of EN

\section{What this paper adds?}

- An early exploration of PICU nurses' decision-making around GRV measurement in a single UK centre

- To provoke further thought and research around this ritualistic nursing practice 


\section{References}

Ahmad S, Kaitha S, Morton J et al (2012) Nasogastric Tube feedings and Gastric Residual Volume: a regional survey. Southern Medical Journal; 105: 394-398.

Bartlett-Ellis R, Fuehne J (2015) Examination of Accuracy in the Assessment of Gastric Residual Volume: a simulated, controlled study. JPEN; 39(4): 434-440.

Bockenkamp B, Jouvet $P$, Arsenault V et al (2009) Assessment of calories prescribed and delivered in critically ill children. e-SPEN 4: e172-e175.

Bollineni D, Minocha A (2011) Nursing Practice of Checking Gastric Residual Volumes based on old dogmas: opportunity to improve patient care while decreasing healthcare costs. J La State Med Soc; 163: 205-209.

Burnard P, Gill P, Stewart E et al. (2008) Analysing and presenting qualitative data. British dental Journal 204(8): 429-432.

Elke G, Felbinger T, Heyland D (2015) Gastric Residual Volume in Critically III Patients: A Dead Marker or Still Alive? Nutrition in Clinical Practice; 30(1): 59-71

Floh A, Slicker J, Schwartz S (2016) Nutrition and Mesenteric Issues in Pediatric Cardiac Critical Care. Ped Crit Care Med doi:10.1079/PCC.0000000000000801

Hodges C and Vincent P (1993) Why do NICU nurses not refeed gastric residuals prior to feeding by gavage? Neonatal Network 12(8): 37-40.

Parker L, Torrazza RM, Yuefeng L et al (2015) Aspiration and Evaluation of Gastric Residuals in the NICU: State of the Science. J Perinatal Neonatal Nurs; 29(1): 51-59.

Kuppinger D, Rittler P, Hartl W et al (2013) Use of gastric residual volume to guide enteral nutrition in critically ill patients: a brief systematic review of clinical studies. Nutrition; $1075-$ 1079

Leong A, Cartwright K, Guerra G et al (2013) A Canadian survey of perceived barriers to initiation and continuation of enteral feeding in PICUs. Ped Crit Care Med; 15: 2

Li YL, Lin HC, Torrazza R et al (2014) Gastric Residual Evaluation in Preterm Neonates: A useful Monitoring technique or a hindrance? Pediatrics \& Neonatology; 55: 335-340.

Marik P (2014) Enteral Nutrition in the Critically Ill: Myths and Misconceptions. Ped Crit Care Med; 42(4): 962-969. 
McClave S, Snider H (2002) Clinical Use of gastric Residual Volumes as a monitor for patients on enteral tube feeding JPEN; 26: S43-S50

McClave S, Lukan J, Steafater C et al (2005) Poor validity of residual volumes as a marker for risk of aspiration in critically ill patients. Crit Care Med; 33: 324-330

Mehta N, McAleer D, Hamilton D et al (2010) Challenges to optimal enteral nutrition in a multidisciplinary pediatric intensive care unit. JPEN; 34: 38-45.

Mehta N, Bechard L, Cahill N et al (2012) Nutritional practices and their relationship to clinical outcomes in critically ill children - an international multicentre cohort study. Crit Care Med; 40: 2204-2211

Mehta N, McAleer D, Hamilton S et al(2010) Challenges to optimal enteral nutrition in a multidisciplinary pediatric intensive care unit. JPEN; 34: 38-45.

Montejo J, Minambres E, Bordeje L et al.(2010) Gastric residual volume during enteral nutrition in ICU patients: the REGANE study. Intensive Care Med; 36:1386-1393.

National Patient Safety Agency (2011) Patient Safety Alert NPSA/2011/PSA002:

Reducing the harm caused by misplaced nasogastric feeding tubes in adults, children and infants. www.nrls.npsa.nhs.uk/EasySiteWeb/getresource.axd?Asset|D=129697 accessed 13 February 2017

Quenot JP, Plantefeve G, Baudel JL et al (2010) Bedside adherence to clinical practice guidelines for enteral nutrition in critically ill patients receiving mechanical ventilation: a prospective, multicentre, observational study. Critical Care; 14:R37

Ozen N, Tosun N, Yamanel L. et al (2016) Evaluation of the effect on patient parameters of not monitoring gastric residual volume in intensive care patients on a mechanical ventilator receiving enteral nutrition: a randomized clinical trial. J Crit Care; 33: 137-144.

Parrish C, McClave S. (2008) Checking Gastric Residual Volumes: A Practice in Search of Science? Practical Gastroenterology; 33-47.

Poulard F, Dimet J, Martin-Lefevre L et al (2010)Impact of not measuring Residual Gastric Volume in Mechanically Ventilated Patients Receiving Early Enteral Feeding: a prospective before-after study JPEN; 34(2): 125-130.

Reignier J, Mercier E, Le Gouge A et al (2013). Effect of not monitoring residual gastric volume on risk of ventilator-associated pneumonia in adults receiving mechanical ventilation and early enteral feeding. JAMA; 309(3):

Tume L, Latten L, Darbyshire A (2010) An evaluation of enteral feeding practices in critically ill children. Nurs in Crit Care; 15: 291-299 
Tume L, Carter B, Latten L (2013) A UK and Irish survey of enteral nutrition practices in paediatric intensive care units. Br J Nutrition; 109:1304-1322.

Tume LN. Allan E, Latten L, Davies S, Lefèvre MH, Nicolas GW, Valla FV (2016). Does routine gastric residual volume measurement impact on calorie delivery in the PICU? Oral free paper presentation at UK PICS Conference 2016, Oct 3-5 Southampton.

Valla F, Gaillard-Le Roux B, Ford-Chessel C et al (2015) NutriRea-Ped 2014: The Nursing Survey on Nutrition Practices in French-speaking Pediatric Intensive Care Units. JPGN doi:10.1097/MPG.000000000000930 


\section{Figure 1 Survey instrument}

1. What is your role on PICU?

Assistant practitioner

Band 5 nurse

Band 6 nurse

Band 7 nurse

2. How many years PICU experience do you have?

3. Do you have a PICU/ICU course?

Yes

No

4. In your opinion what is the potential harm from high gastric aspirates?

5. How frequently do you usually measure gastric aspirates in PICU?

6. What are your reasons for measuring gastric aspirates?

To check feed tolerance

To check/confirm naso-gastric tube position

Other please state

7. What factors affect your decision to DISCARD aspirates during enteral feeding on PICU? Please rank the answers below in order of importance to you eg $10=$ most important to 1 not important

The amount (volume) obtained

If the aspirate looks undigested

The colour of the aspirate

The condition of the child

8. What factors affect your decision to REPLACE gastric aspirates?

Please rank the answers below in order of importance to you eg 10= most important to 1

not important

The amount (volume) obtained.

How digested the aspirate looks.

The colour of the aspirate

The condition of the child.

9. If you decide to withhold feeds based on the gastric aspirate, what factors do you base this decision on?

10. In what time frame would you restart feeds? What factors affect your decision?

11. In your opinion what are the biggest barriers to delivering adequate volumes of enteral feed on PICU? 
12. On a scale of 1 to 10 , how important do you think the nurse's role is in STARTING enteral feeding?

Please rate from $1=$ not important to10 = very important .

13. On a scale of 1 to 10 , how important do you think the nurse's role is in DELIVERING (giving the feed) enteral feeding?

Please rate from 1= not important to10 = very important.

14. On a scale of 1 to 10 how important do you think the nurse's role is in EVALUATING enteral feeding?

Please rate from 1= not important to10 = very important.

15. Are you familiar with the XXXXX PICU guidelines around enteral feeding and gastric aspirates?

Yes

No

Other (please specify)

16. If yes, do you know what guidance they give around acceptable gastric aspirates and returning aspirates? Please write this below

17. How would you feel about NOT measuring gastric aspirates routinely?

Very worried

Worried

OK

Happy

Very happy

18. What would be your concerns about NOT measuring gastric aspirates?

19. If you could not assess gastric aspirate what would you use to assess feed 'tolerance'?

20. How would you feel about being part of a UK wide study where gastric aspirates were NOT measured compared to standard care (where gastric aspirates were routinely measured)?

21. Are there any other comments you would like to make regarding enteral feeding and gastric aspirates on PICU? 
Figure 2 Nurses' perceived rating of the importance their role in enteral nutrition

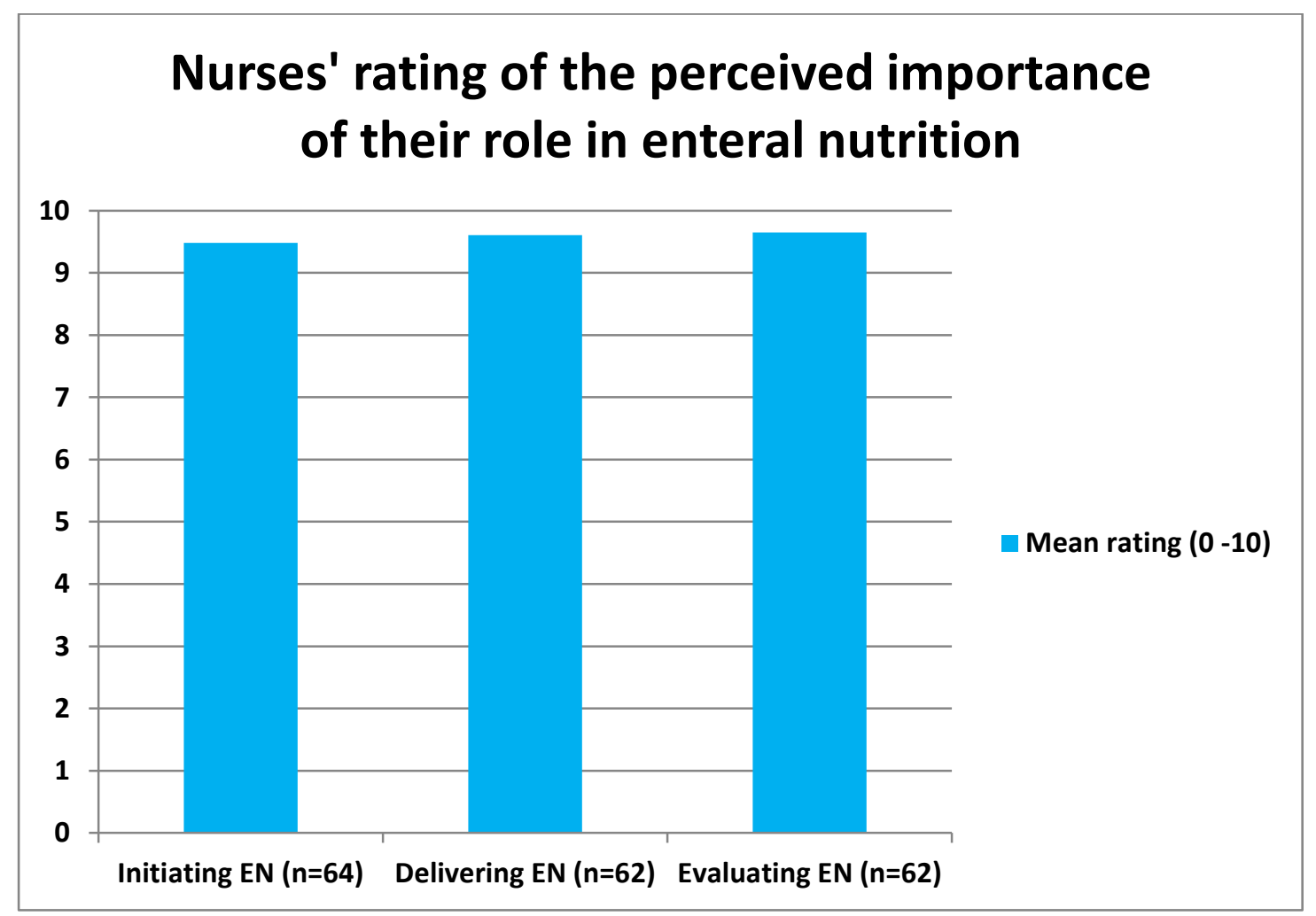

Likert scale $0-10(0=$ not important to $10=$ very important $)$ EN: Enteral Nutrition

$\mathrm{n}=$ Responses per question 


\section{Table 1}

PICU Nurses' perceived harms from high Gastric Residual Volume (GRV)

\begin{tabular}{|l|l|}
\hline Nurses' perceived harms & $\%(\mathrm{~N})$ \\
\hline Risk of aspiration & $44 \%(40 / 90)$ \\
\hline Malabsorption of enteral feeds & $20 \%(18 / 90)$ \\
\hline Risk of vomiting & $19 \%(17 / 90)$ \\
\hline Abdominal distention & $10 \%(9 / 90)$ \\
\hline Inadequate nutrition & $6.6 \%(6 / 90)$ \\
\hline Miscellaneous reasons & $6.6 \%(6 / 90)$ \\
\hline Abdominal discomfort & $5.5 \%(5 / 90)$ \\
\hline Necrotising enterocolitis (NEC) & $2.2 \%(2 / 90)$ \\
\hline Poor weight gain & $2.2 \%(2 / 90)$ \\
\hline
\end{tabular}


Figure 3 Alternative indicators nurses reported they would use to assess feed tolerance without Gastric Residual Volume (GRV)

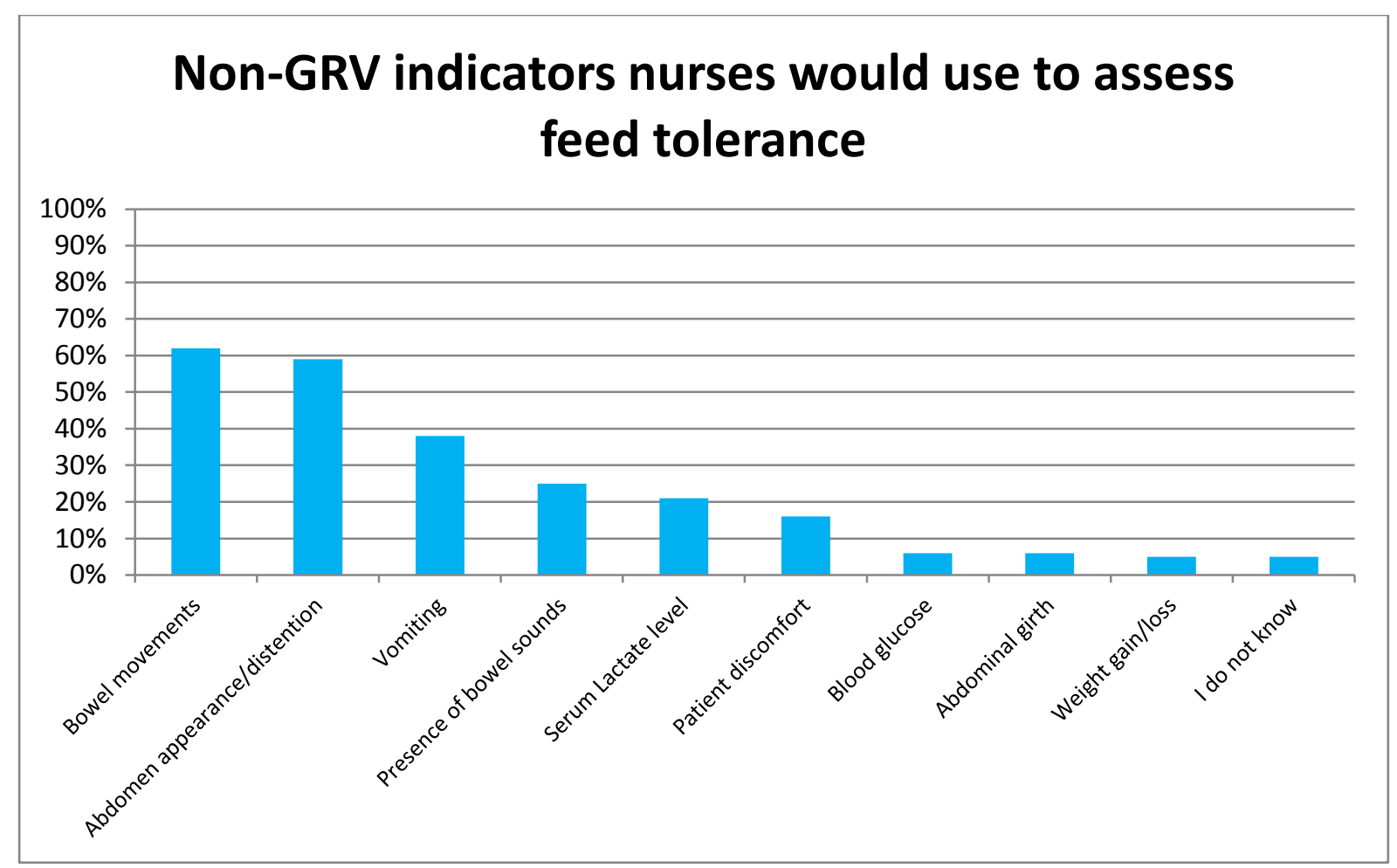

